\title{
Generalized skyrmions in QCD and the electroweak sector
}

\author{
John Ellis, ${ }^{a, b}$ Marek Karliner ${ }^{c}$ and Michal Praszalowicz ${ }^{d}$ \\ ${ }^{a}$ Theoretical Particle Physics and Cosmology Group, Physics Department, \\ King's College London, London WC2R 2LS, U.K. \\ ${ }^{b}$ TH Division, Physics Department, CERN, \\ CH-1211 Geneva 23, Switzerland \\ ${ }^{c}$ Raymond and Beverly Sackler School of Physics and Astronomy, \\ Tel Aviv University, Israel \\ ${ }^{d}$ M. Smoluchowski Institute of Physics, Jagiellonian University, \\ 4 Reymonta Str., 30-059 Kraków, Poland \\ E-mail: John.Ellis@cern.ch, marek@neutron.tau.ac.il, \\ michal@if.uj.edu.pl
}

ABSTRACT: We discuss the stability and masses of topological solitons in QCD and stronglyinteracting models of electroweak symmetry breaking with arbitrary combinations of two inequivalent Lagrangian terms of fourth order in the field spatial derivatives. We find stable solitons for only a restricted range of the ratio of these combinations, in agreement with previous results, and we calculate the corresponding soliton masses. In QCD, the experimental constraints on the fourth-order terms force the soliton to resemble the original Skyrmion solution. However, this is not necessarily the case in strongly-interacting models of electroweak symmetry breaking, in which a non-Skyrmion-like soliton is also possible. This possibility will be constrained by future LHC measurements and dark matter experiments. Current upper bounds on the electroweak soliton mass range between 18 and $59 \mathrm{TeV}$, which would be reduced to 4.6 to $8.1 \mathrm{TeV}$ with the likely sensitivity of LHC data to the fourth-order electroweak Lagrangian parameters.

Keywords: Higgs Physics, Beyond Standard Model, Technicolor and Composite Models ARXIV EPRINT: 1209.6430 


\section{Contents}

1 Introduction 1

2 Classical mass and stability of an SU(2) soliton 3

3 Phenomenological estimates of soliton masses $\quad 8$

3.1 QCD baryons 9

3.2 Current bounds on electroweak baryon masses 11

$\begin{array}{ll}3.3 & \text { Prospective LHC bounds on electroweak baryon masses } \\ \end{array}$

4 Electroweak baryons as dark matter? $\quad 12$

5 Concluding remarks $\quad 14$

\section{Introduction}

It is a generic feature of strongly-interacting theories that their vacua are not invariant under all their global symmetries, and that the resulting effective low-energy Lagrangians possess soliton solutions $[1,2]$. The archetype is QCD, and many other four-dimensional examples have been studied theoretically (for a rigorous example, see $[3,4]$ ). In the case of QCD, most studies have built upon the pioneering work of Skyrme [5, 6], and Skyrmion phenomenology has had a number of phenomenological successes [7-13]. However, generalizations of the Skyrme model are possible even in QCD, and a fortiori in other stronglyinteracting models that may exhibit dynamics rather different from QCD.

Strongly-interacting models of electroweak symmetry breaking have long attracted considerable attention $[14,15]$, with renewed intensity now that LHC experiments have discovered a Higgs-like particle [16, 17]. However, although it has some of the characteristic properties predicted for the Higgs boson in the Standard Model, the jury is still out, and many alternative scenarios described by low-energy effective chiral Lagrangians remain viable [18-21]. For example, there is active interest in the simplest possibility that the recently-discovered particle might be the pseudo-dilaton of some nearly-conformal stronglyinteracting electroweak sector [22-43]. Discriminating between scenarios for electroweak symmetry breaking is a phenomenological priority, and the existence (or otherwise) of soliton solutions may be a valuable diagnostic tool for this task [44].

The masses and other properties of solitons in models described by effective low-energy chiral Lagrangians depend on the strengths of higher-order terms in their derivative expansions, and specifically of the coefficients of the fourth-order terms [2]. In the case of the minimal effective Lagrangian for $\mathrm{SU}(2) \times \mathrm{SU}(2) \rightarrow \mathrm{SU}(2)$ that may be used to describe both QCD and electroweak symmetry breaking, there are two such parameters, as 
discussed in section 2. One of these was considered originally by Skyrme [5, 6], and has been the basis for most subsequent studies of Skyrmion phenomenology [7-11]. However, $a$ priori the other term could also be present, and there have been some studies of generalized soliton solutions in the presence of this extra term [45-50]. In generic strongly-interacting models there may be a relation between the fourth-order Lagrangian parameters and the electroweak S parameter, but exploring this goes beyond the scope of this paper.

Truncating the derivative expansion at fourth order is dictated primarily by practical reasons, namely that phenomenological information is available only about the fourth-order electroweak coefficients. However, we recall that the contributions of higher-derivative terms to solitonic masses are not parametrically suppressed [51]. In the leading order of the $1 / N_{\mathrm{c}}$ expansion the soliton mass can be written as an expansion in powers of the soliton size $r_{0}$ :

$$
M \sim 4 \pi \bar{v} N_{\mathrm{c}}\left[a_{2} \bar{v} r_{0}+a_{4} \frac{1}{\bar{v} r_{0}}+a_{6} \frac{1}{\left(\bar{v} r_{0}\right)^{3}}+\ldots\right],
$$

where $\bar{v}=v / \sqrt{N_{\mathrm{c}}}$ is the $N_{\mathrm{c}}$-independent energy scale of chiral symmetry breaking (corresponding to $F_{\pi} / \sqrt{N_{\mathrm{c}}}$ in QCD), and the $a_{n}$ are generic coefficients of $n$ 'th-order terms in the derivative expansion that are expected to be of order 1 . If one minimizes just the first two terms of $M$ with respect to $r_{0}$, treating the sixth-order term as a perturbation, its contribution is linear in $a_{6}$. This might suggest that for large $a_{6}$ (and this is the case in QCD, where a sixth-order term is generated by $\omega$-meson exchange $[12,52,53])$ the sixth-order term would even dominate. However, if one minimizes the whole expression for $M$ (up to a given order) the situation changes dramatically. For positive $a_{6}$, the sixth-order term provides a 'barrier' around $r_{0}=0$ (as do higher-order terms). Consequently, the optimal $r_{0}$ is increased and, although the $a_{2} \bar{v} r_{0}$ term increases, the 4 -th and 6 -th order terms get smaller. As a result the sensitivity of the soliton mass to the 6 -th order term saturates at large $a_{6}>0$. The soliton mass with large positive $a_{6}$ is obviously larger than without it, but "only" by factor $\sim 2$ [53], so higher-order terms need not have large effects.

One should also remember that keeping only one specific fourth-order term, namely the Skyrme term introduced in section 2, gives results accurate to within $20-30 \%$ in the case of QCD, which would be sufficient for our purposes here. Estimates using the Skyrme term alone actually overshoot the experimental nucleon mass, and we find that a somewhat larger range of classical masses is obtained when the non-Skyrme term is included. We infer that the truncated Lagrangian may well be a useful guide to possible upper limits on electroweak soliton masses.

The classical approximation is then followed by semiclassical quantization. Semiclassical rotations are suppressed in QCD in the $1 / N_{\mathrm{c}}$ expansion [2]. So even if a given Lagrangian generates higher-order time derivatives, which is the case for the solitons with the non-Skyrme term present, they can be neglected within large $N_{\mathrm{c}}$ approximation. A similar justification is applicable in many strongly-interacting models of electroweak symmetry breaking, but needs exploration on a case-by-case basis. Here we do not study issues beyond the classical approximation. Nor do we consider the negative Casimir $\mathcal{O}\left(N_{\mathrm{c}}^{0}\right)$ contribution to the soliton mass, which is the hardest to calculate. One can find discussion and estimates of the Casimir energy in the QCD Skyrme model in refs. [54, 55]. 
As we review later in the context of our analysis, the existence or absence of stable soliton solutions hinges upon the ratio of the two fourth-order coefficients, and there is a generic range of this ratio where no stable solitons exist [45-50]. Equally, there is a generic range, including the original Skyrmion as a special case, where solitons do exist and are stable, at least against spherically-symmetric decay. In the case of QCD, large- $N_{c}$ arguments favour values of the fourth-order parameters within this stable range, close to the Skyrmion limit, and this possibility is also favoured by the available phenomenological estimates of these parameters in the effective chiral Lagrangian of QCD [56, 57].

However, currently we know very little about the possible magnitudes of the fourthorder coefficients in the electroweak case, and it is possible that their ratio is quite unlike the original Skyrme model, quite possibly in the range where no stable solitons exist. On the other hand, if the strongly-interacting electroweak sector is based on a theory with underlying constituents that bind to form 'electroweak baryons', one would expect solitons to exist and describe qualitatively their masses and other properties, even if they are rather different from the baryons and Skyrmions in QCD-like theories [44].

The purpose of this paper is to explore the non-Skyrmionic possibilities [45-50], particularly in the electroweak case, discussing their existence, stability and masses in the classical approximation. This analysis is an essential ingredient in the exploration of the consistency of different strongly-interacting electroweak models with experiment. To this end, we assess the prospects for probing strongly-interacting electroweak models by confronting present $[58,59]$ and future limits [58] on (measurements of) fourth-order interaction parameters with limits on (measurements of) electroweak baryon masses. We also note that stable solitons would be present at some level in the Universe today as relics from the Big Bang and contribute to the dark matter [60]. One should check whether calculations of their abundance are compatible with cosmological and astrophysical estimates of the density of cold dark matter, and whether estimates of the rate for their elastic scattering on nuclei [61] are compatible with upper limits from direct searches for dark matter [63].

The structure of this paper is as follows. In section 2 we discuss the mass and stability of an $\mathrm{SU}(2)$ soliton in the classical limit, including both possible fourth-order derivative terms. These calculations are used in section 3 to set phenomenological bounds on the possible masses of QCD and electroweak solitons, taking into account the present and prospective experimental constraints on the fourth-order terms. Section 4 summarizes considerations concerning electroweak solitons as cold dark matter, and section 5 contains some concluding remarks.

\section{Classical mass and stability of an SU(2) soliton}

The effective chiral Lagrangian corresponding to some strongly-interacting sector with spontaneously-broken $\mathrm{SU}(2) \times \mathrm{SU}(2)$ chiral symmetry is usually organized in terms of (even) powers of derivatives of the chiral field:

$$
U(x)=\exp \left(i \frac{\vec{\tau} \cdot \vec{\pi}(x)}{v}\right),
$$


where the fields $\vec{\pi}(x)$ correspond to the Nambu-Goldstone bosons (pions in the case of QCD, longitudinal polarization states of massive gauge bosons in the case of a strongly-interacting electroweak sector) and $v$ is a typical symmetry breaking scale $\left(v=F_{\pi}=93 \mathrm{MeV}\right.$ in QCD, $v \sim 246 \mathrm{GeV}$ in the case of electroweak theory). The derivative expansion is usually truncated at the fourth order, which could be reliable at energies below the characteristic strong-interaction scale, $\sim \mathrm{GeV}$ in $\mathrm{QCD}$ and $\sim \mathrm{TeV}$ or more in the electroweak theory:

$$
\mathcal{L}_{\text {eff }}=\mathcal{L}_{2}+\mathcal{L}_{4}
$$

where

$$
\mathcal{L}_{2}=\frac{v^{2}}{4} \operatorname{Tr}\left(\partial_{\mu} U \partial^{\mu} U^{\dagger}\right)
$$

In the $\mathrm{SU}(2)$ case there are two independent invariants containing just four space-time derivatives $[45-50]$ :

$$
\mathcal{L}_{4}=2 s \operatorname{Tr}\left[\left(R_{\mu} R_{\nu}\right)\left(R^{\mu} R^{\nu}\right)-\left(R_{\mu} R^{\mu}\right)^{2}\right]+2 t \operatorname{Tr}\left[\left(R_{\mu} R_{\nu}\right)\left(R^{\mu} R^{\nu}\right)+\left(R_{\mu} R^{\mu}\right)^{2}\right],
$$

where

$$
R_{\mu}=\partial_{\mu} U U^{\dagger}
$$

The parameters $s$ and $t$ can be in principle calculated from the underlying stronglyinteracting theory by integrating out the constituent degrees of freedom (quarks and gluons in the case of QCD, and yet to be determined in an electroweak theory) or - in a phenomenological approach - can be extracted from the data on the scattering of the Nambu-Goldstone bosons or massive gauge bosons [58]. ${ }^{1}$ In QCD, as we discuss in more detail later, the large- $N_{\mathrm{c}}$ expansion and pion data indicate that $|t| \ll|s|$, so that the effective Lagrangian contains (in a first approximation) only the kinetic term (2.3) and the first term in (2.4), as in Skyrme's original work. For this reason, we refer to this as the Skyrme term, and refer to other term in (2.4), that with coefficient $t$, as the non-Skyrme term.

Over fifty years ago already, Skyrme [5,6] observed that (2.2) with $t=0$ possesses solitonic solutions that, due to the fact that $\pi_{3}(\mathrm{SU}(2))=\mathbb{Z}$, carry an integer-valued topological quantum number

$$
B=\frac{1}{24 \pi^{2}} \int d^{3} x \epsilon^{i j k} \operatorname{Tr}\left[\left(U^{\dagger} \partial_{i} U\right)\left(U^{\dagger} \partial_{j} U\right)\left(U^{\dagger} \partial_{k} U\right)\right]
$$

that can be interpreted as baryon number (see also [1]).

It is straightforward to calculate the classical contribution to the mass of the baryon in terms of the parameters $v, s$ and $t$. In principle, all terms in the derivative expansion make significant contributions to the soliton mass, whereas we have no information on the possible magnitudes of higher-order terms. On the other hand, we know that classical calculations in QCD keeping only the fourth-order term Skyrme term, i.e., setting $t=0$, are accurate to $\sim 20-30 \%[7-11,13]$. In the case with non-zero non-Skyrme term a somewhat larger masses are allowed, cf. our calculation below for QCD point A in Sect 3.1.

\footnotetext{
${ }^{1}$ For rigorous lower bounds from Lorentz invariance, analyticity, unitarity and crossing, see [59].
} 
We therefore hope that classical calculations in the electroweak case, keeping the general form of fourth-order coupling, sample the range of possible masses with similar accuracy.

Possible refinements to this classical calculation could include calculation of the Casimir energy, inclusion of the current-quark mass terms and semiclassical quantization of the rotational modes (which provide better agreement with the observed baryon masses). However, we do not go into them here, for three reasons in addition to our ignorance of possible higher-order terms in the electroweak case. One is that we are primarily interested in bounds on the masses of the possible electroweak baryons, rather than in details of their spectrum. A second reason is that, in any case, we lack the information about the underlying strongly-interacting theory that would be needed to calculate the non-classical corrections in this case. The third reason is that in reality, even in QCD, the parameter $t$ is not necessarily negligible. For example in an approximation where the Skyrme Lagrangian is derived by integrating out heavy mesons (typically $\rho$ and $\sigma$ ) one can relate the parameters $s$ and $t$ to the heavy meson masses [12, 53, 62]. Comparing our Lagrangian (2.2) with ref. [62] we get:

$$
s=\frac{F_{\pi}^{2}}{48} \frac{3 m_{\sigma}^{2}-2 m_{\rho}^{2}}{m_{\rho}^{2} m_{\sigma}^{2}}=(0.6 \div 3.2) \times 10^{-4}, \quad t=\frac{F_{\pi}^{2}}{24 m_{\sigma}^{2}}=(5.8 \div 8.4) \times 10^{-4}
$$

with $F_{\pi}=93 \mathrm{MeV}$. The soliton is unstable with these values, as we discuss shortly in connection with figure 1 , since $t / s>1.7$ and stable solutions exist only for $t / s \leq 0.29$. Moreover, the values (2.7) are beyond the range allowed for the QCD effective Lagrangian discussed in section 3.1. Therefore, it does not seem possible to constrain the electroweak $s$ and $t$ parameters using ideas about the (as yet unknown) technimeson masses. ${ }^{2}$

Our primary interest here will be the classical soliton mass in the presence of a nonnegligible non-Skyrme term, and how the possibility that $t \neq 0$ affects the (approximate) mass bounds provided by the Skyrme calculation with $t=0$ discussed in [44].

Classical soliton solutions of the chiral field equations are usually found within the spherically-symmetric 'hedgehog' Ansatz for a static field configuration:

$$
U(\vec{r})=\exp \left(i \frac{\vec{\tau} \cdot \vec{r}}{r} P(r)\right)
$$

where the profile function $P(r)$ is a solution to the Euler-Lagrange equation of motion, which for the Lagrangian (2.2)-(2.4) takes the following form:

$$
P^{\prime \prime}(\rho)=-\frac{G(\rho)}{F(\rho)}
$$

with boundary conditions

$$
P(0)=\pi, P(\infty)=0
$$

\footnotetext{
${ }^{2}$ We note also that (2.7) implies $t>0$, whereas the bounds shown in figure 4 do not exclude negative $t$.
} 
Here

$$
\begin{aligned}
F(\rho) \equiv & \rho^{2}\left(1-96 t P^{\prime 2}\right)+64 s \sin ^{2} P, \\
G(\rho) \equiv & -64 t \rho P^{\prime 3}+32 s \sin 2 P P^{\prime 2}+2 \rho P^{\prime} \\
& -\sin 2 P\left(1+32(s-t) \frac{\sin ^{2} P}{\rho^{2}}\right),
\end{aligned}
$$

and the dimensionless radial variable $\rho$ is defined as

$$
\rho \equiv r v
$$

where $v=F_{\pi}, \simeq 246 \mathrm{GeV}$ in the QCD and electroweak cases, respectively.

Following the pioneering paper [2], classical solutions of the field equations were studied numerically long ago in $[45,46]$ and stability regions in the $(s, t)$ parameter space were analyzed in [47-50]. We have repeated their analyses for the purpose of the present work with the following results.

The total classical soliton mass

$$
M_{\mathrm{sol}}=4 \pi v\left(M_{2}+M_{4}\right)
$$

where

$$
\begin{aligned}
& M_{2}=\frac{1}{2} \int_{0}^{\infty} d \rho\left(\rho^{2} P^{\prime 2}+2 \sin ^{2} P\right), \\
& M_{4}=16 s \int_{0}^{\infty} d \rho\left(2 P^{\prime 2} \sin ^{2} P+\frac{\sin ^{4} P}{\rho^{2}}\right)-8 t \int_{0}^{\infty} d r\left(\rho^{2} P^{\prime 4}+2 \frac{\sin ^{4} P}{\rho^{2}}\right),
\end{aligned}
$$

where the contribution $M_{2}$ corresponds to $\mathcal{L}_{2}$ and is clearly always positive, and the contribution $M_{4}$ corresponds to $\mathcal{L}_{4}$. By changing variable once more: $\rho \rightarrow \rho / \sqrt{|s|}$ or $\rho \rightarrow \rho / \sqrt{|t|}$, one can show that the solutions depend only on the ratio $t / s$. By rescaling: $\rho \rightarrow \lambda \rho$, one can show that solutions exist only when $M_{4}$ is positive. Inspecting directly $M_{4}$ in (2.14), we see that in the fourth quadrant of the $(s, t)$ plane $(s>0, t<0) M_{4}$ is always positive, so that solutions of (2.9) always exist, whereas in the second quadrant $(s<0, t>0) M_{4}$ is always negative, so there are no solutions of (2.9). In the first and third quadrants positivity bounds can be derived [45-47]. It turns out that in the first quadrant $M_{4}$ is negative for $t / s>2$, so the soliton is unstable, and in the third quadrant $M_{4}$ stays positive for $t / s>2$, so the soliton is stable. This is illustrated in figure 1, where the regions of positive and negative $M_{4}$ are displayed by green shading (diagonal squares) and red shading (vertical squares), respectively.

In the remaining parts of quadrants $I$ and $I I I$, no positivity bounds can be derived and the positivity of $M_{4}$ has to be checked numerically. It turns out that in the third quadrant $M_{\text {sol }}>0$ and $\rightarrow 0^{+}$when $t / s \rightarrow 2^{+}$, whereas no solution exists for $t / s<2$. The situation is different in the first quadrant, where solutions with positive $M_{4}$ exist for small 


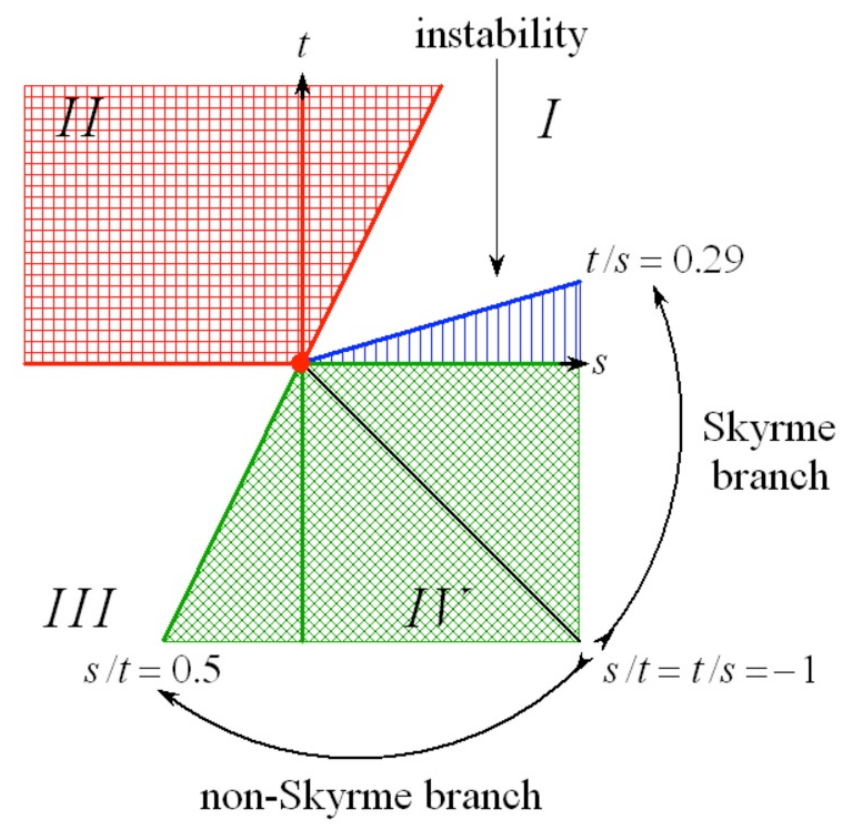

Figure 1. The $(s, t)$ parameter plane, indicating with green shading (diagonal squares) the range of parameters satisfying the positivity bound $M_{4}>0$ and hence admitting a solution of the differential equation (2.9), and with red shading (vertical squares) the range where solutions do not exist due to the negativity of the fourth order term: $M_{4}<0$. In the range $t / s<0.29$ within the first quadrant, indicated in blue (vertical bars), solutions with positive $M_{4}$ exist, but no positivity bound can be derived.

$t / s$ below the line $t / s \simeq 0.29$, where the function $F(\rho)$ vanishes and equation (2.9) cannot be solved. Interestingly, $F(\rho)$ is equal to the second variation of $M_{\text {sol }}$, so the positivity of $F(\rho)$ is a necessary condition for $M_{\text {sol }}$ to be the minimum with respect to the variations of $P(r)$. Below the line $t / s \sim 0.29$ the solution is classically stable, and above this line it is classically unstable.

However, it has been shown in $[45,46]$ that there is no lower limit on the soliton mass anywhere in the first quadrant. This means that, when $t / s<0.29$, the classical solution discussed above, though stable against local spherically-symmetric perturbations, can only be metastable at best, and might be unstable against non-spherically-symmetric perturbations. This region is shaded blue (with vertical bars) in figure 1 .

As already remarked, soliton solutions depend only on the ratio $t / s$ (or $s / t$ ). We analyze solutions starting from the Skyrme-like case with $t=0$ and the non-Skyrme-like case with $s=0$, and have divided the allowed parameter space into two branches on either side of the line $t / s=-1$, as also depicted in figure 1. In order to solve equation (2.9) within the Skyrme branch where $s \neq 0$, we have rescaled $\rho \rightarrow \rho / \sqrt{s}$ and evaluated the soliton mass in units of $4 \pi v \sqrt{s}$, whereas within the non-Skyrme branch where $t \neq 0$ we have rescaled $\rho \rightarrow \rho / \sqrt{-t}$ and evaluated the soliton mass in units of $4 \pi v \sqrt{-t}$. The results are presented in figure 2 as functions of $\varepsilon \equiv t / s$ for the Skyrme branch and $\varepsilon \equiv s / t$ for the non-Skyrme branch. 


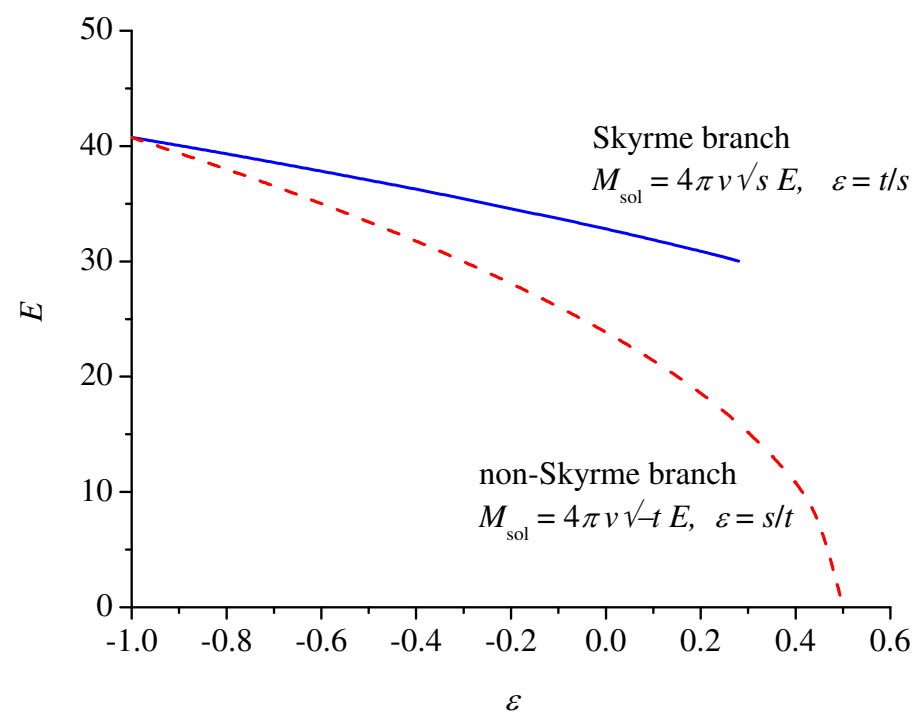

Figure 2. The soliton mass in units of $4 \pi v \sqrt{s}$ for the Skyrme branch where $\epsilon=t / s$ (solid blue line), and for the the non-Skyrme branch in units of $4 \pi v \sqrt{-t}$ where $\epsilon=s / t$ (dashed red line). The Skyrme branch ends at $t / s \sim 0.29$ (see figure 1 ).

Finally, in figure 3 we present a contour plot of the classical soliton mass in units of $4 \pi v$ over the allowed range of parameter space. We see that the contours of constant $M_{\text {sol }}$ are almost parallel to the line $t / s=2$ where the soliton mass vanishes. This feature is very helpful for finding the maximum of the generalized soliton mass, as we discuss in the next section.

\section{Phenomenological estimates of soliton masses}

We now discuss constraints on the conventional baryon mass using constraints on the parameters of the low-energy effective chiral Lagrangian for QCD, and constraints on the masses of possible 'electroweak baryons' given by the constraints on higher-order electroweak Lagrangian parameters from electroweak theory, current phenomenology and the potential sensitivity from the LHC $[44,58]$.

Phenomenological constraints on higher-order Lagrangian parameters are often given in terms of the coefficients $\alpha_{4,5}$ that are related to the parameters $s$ and $t$ discussed in section 2 in the following way [44]:

$$
s=\frac{\alpha_{4}-\alpha_{5}}{4}, t=\frac{\alpha_{4}+\alpha_{5}}{4} .
$$

Although limits on electroweak baryon masses are our principal interest, we first discuss the limits that can be derived for ordinary baryons in QCD, comparing the non-Skyrmion case with $t \neq 0$ with the conventional Skyrmion case $t=0$. 


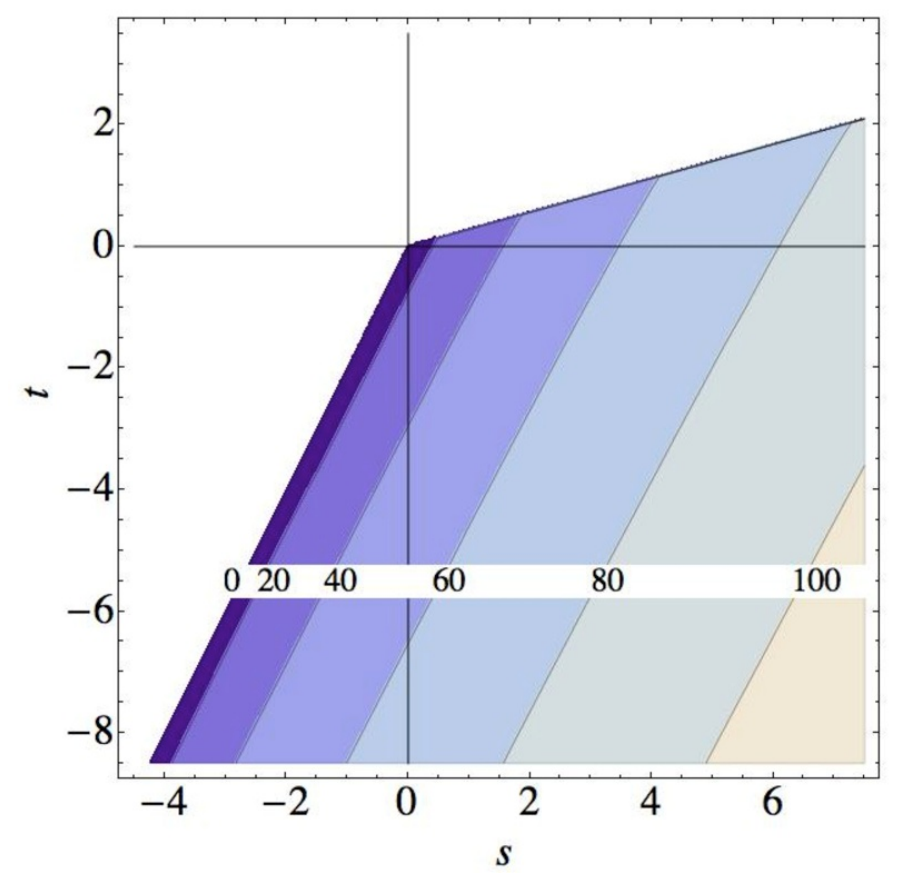

Figure 3. Contour plot of the mass of the generalized Skyrmion in units of $4 \pi v$.

\subsection{QCD baryons}

Higher-order coefficients in the low-energy effective Lagrangian of QCD have been the subject of many studies. Here we use the following ranges extracted from low-energy strong-interaction data: $[44,56,57]$ :

$$
\begin{aligned}
& 11 \times 10^{-4}<\alpha_{4}<17 \times 10^{-4}, \\
& 14 \times 10^{-4}<\alpha_{4}-\alpha_{5}<40 \times 10^{-4} .
\end{aligned}
$$

We can compare these values with the predictions of the large- $N_{\mathrm{c}}$ approximation within the framework of chiral $\mathrm{SU}(3) \times \mathrm{SU}(3) \rightarrow \mathrm{SU}(3)[57]:$

$$
\begin{aligned}
& \alpha_{4}=18 \times 10^{-4}, \\
& \alpha_{5}=-16 \times 10^{-4} .
\end{aligned}
$$

The bounds (3.2) are displayed in figure 4, where the large- $N_{\mathrm{c}}$ prediction is also indicated, as a red spot. ${ }^{3}$

As already discussed in section 2 the contours of constant $M_{\text {sol }}$ are almost parallel to the line $t=2 s$. Therefore the maximal mass corresponds to the right-most corner of the region allowed by the QCD bounds, called Point A below. For completeness, we also include two pure Skyrme points (B and $\mathrm{C}$ ) and the point corresponding to minimal mass, located right below the stability line $t / s=0.29$ (Point D):

\footnotetext{
${ }^{3}$ We represent the constraints (3.2), (3.6),$(3.7)$ as parallelograms in the $(s, t)$ plane, whereas they should be ellipses. However, the correlations between the errors in $s$ and $t$ are not available, so these ellipses cannot be drawn accurately.
} 


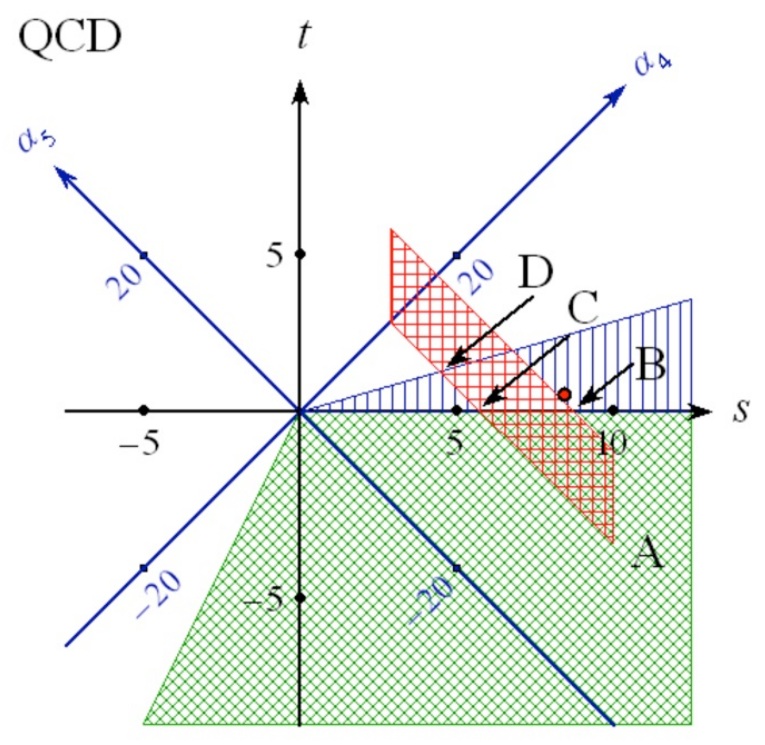

Figure 4. Comparison of the parameter ranges allowed for the low-energy chiral perturbation theory coefficients in QCD [56, 57], eq. (3.2), with the range of parameters where stable baryonic solitons exist. The parameters $\alpha_{4}, \alpha_{5}, s$ and $t$ are in units of $10^{-4}$. The red spot corresponds to the large- $N_{\mathrm{c}}$ values given in eq. (3.3). Point $\mathrm{A}$ has the maximal mass overall, Points B and C bracket the range of parameters allowed in the pure Skyrme limit: $t=0$, and Point D lies on the stability boundary $t / s=0.29$ and has the minimal mass overall.

- A : $(s, t)=(10,-4.5) \times 10^{-4}($ maximal mass point overall $): M_{\mathrm{A}} \simeq 1354 \mathrm{MeV}$;

- $\mathrm{B}:(s, t)=(8.5,0.0) \times 10^{-4}$ (maximal mass Skyrme point): $M_{\mathrm{B}} \simeq 1118 \mathrm{MeV}$;

- $\mathrm{C}:(s, t)=(5.5,0.0) \times 10^{-4}$ (minimal mass Skyrme point) $: M_{\mathrm{C}} \simeq 900 \mathrm{MeV}$;

- $\mathrm{D}:(s, t)=(4.3,1.2) \times 10^{-4}($ minimal mass point overall $): M_{\mathrm{D}} \simeq 728 \mathrm{MeV}$.

We see that the range of classical masses allowed in the Skyrme case $t=0$ includes the physical value of the nucleon mass. In terms of the conventional representation

$$
s=\frac{1}{32 e^{2}},
$$

this range corresponds to

$$
6<e<7.5
$$

We also note that the range of possible QCD baryon masses is extended significantly in the presence of a non-Skyrme term, by $\mathcal{O}(200) \mathrm{GeV}$ in either direction. However, detailed QCD baryon phenomenology including the evaluation of corrections to the classical soliton mass due to the Casimir energy, inclusion of the current-quark mass terms and semiclassical quantization of the rotational modes lies beyond the scope of this paper. 


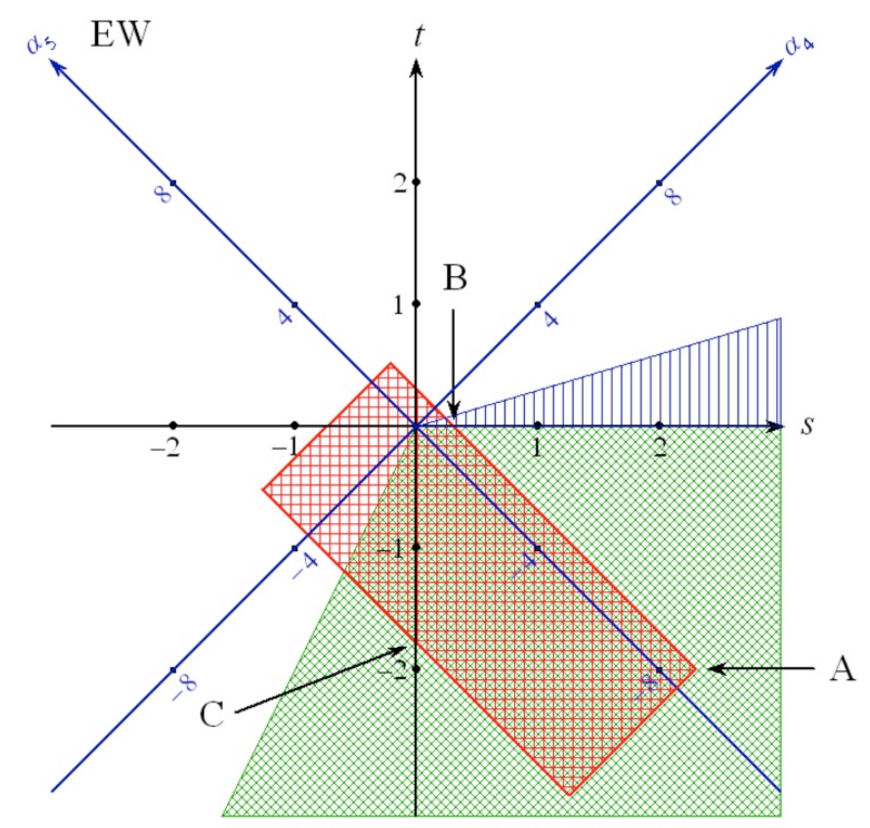

Figure 5. Comparison of the parameter ranges currently allowed [58] for the effective low-energy electroweak Lagrangian with the range of parameters where stable electroweak baryonic solitons exist. The parameters $\alpha_{4}, \alpha_{5}, s$ and $t$ are in units of $10^{-1}$. Point $\mathrm{A}$ has the maximal mass overall, Point $\mathrm{B}$ has the maximal mass allowed in the pure Skyrme limit: $t=0$, and Point $\mathrm{C}$ has the maximal mass allowed in the limit where the Skyrme term vanishes: $s=0$.

\subsection{Current bounds on electroweak baryon masses}

The higher-order coefficients in an effective electroweak Lagrangian are currently poorly constrained, namely by the following bounds [58]:

$$
\begin{aligned}
& -3.5 \times 10^{-1}<\alpha_{4}<0.6 \times 10^{-1}, \\
& -8.7 \times 10^{-1}<\alpha_{5}<1.5 \times 10^{-1} .
\end{aligned}
$$

These constraints are superimposed on the $(s, t)$ plane in figure 5 . As in the QCD case, we superpose various points that illustrate the range of possible electroweak baryon masses currently allowed, as obtained using the values of the soliton mass calculated in section 2 and displayed in figure 2. We note that there is no minimal electroweak baryon mass, since the constraints (3.6) include the possibility that $M_{\mathrm{sol}}=0$ for $t=2 s<0$. The points displayed include the maximal mass point denoted by A, the maximal-mass Skyrme case (Point B), and the maximal-mass case with vanishing Skyrme term $s=0$ (Point C):

- A : $(s, t)=(0.23,-0.20) \quad$ (maximal mass point overall): $M_{\mathrm{A}} \simeq 59 \mathrm{TeV}$;

- $\mathrm{B}:(s, t)=\left(\begin{array}{ll}0.03, & 0.0\end{array}\right)$ (maximal mass Skyrme point): $M_{\mathrm{B}} \simeq 18 \mathrm{TeV}$;

- $\mathrm{C}:(s, t)=(0.0,-0.175)$ (maximal mass with $s=0): \quad M_{\mathrm{C}} \simeq 31 \mathrm{TeV}$. 
This analysis indicates that the upper bound on the possible electroweak baryon mass over the range of parameter space currently allowed is approximately $60 \mathrm{TeV} .{ }^{4}$ This upper limit is somewhat higher than in [44], because we have extended the analysis to the case of a non-zero non-Skyrme term. If the conjectural strongly-interacting electroweak theory were to resemble $\mathrm{QCD}$, i.e., the parameter $t$ were small, the upper limit on the possible electroweak baryon mass limit would be significantly reduced to $\simeq 18 \mathrm{TeV}$.

\subsection{Prospective LHC bounds on electroweak baryon masses}

A study has been made of the prospective LHC sensitivity to the higher-order electroweak Lagrangian terms. It was estimated that, in the absence of a signal, the LHC could yield the following allowed ranges [58]

$$
\begin{gathered}
-7.7 \times 10^{-3}<\alpha_{4}<15 \times 10^{-3}, \\
-12 \times 10^{-3}<\alpha_{5}<10 \times 10^{-3} .
\end{gathered}
$$

These ranges are superimposed on the $(s, t)$ plane in figure 6 . As in the previous examples, we list below the classical masses calculated for three illustrative points that are also displayed in figure 6 :

- A : $(s, t)=(6.75,0.75) \times 10^{-3}($ maximal mass point $): M_{\mathrm{A}} \simeq 8.1 \mathrm{TeV}$;

- $\mathrm{B}:(s, t)=\left(\begin{array}{ll}6.0, & 0.0\end{array}\right) \times 10^{-3}$ (Skyrme point): $\quad M_{\mathrm{B}} \simeq 7.9 \mathrm{TeV}$;

- $\mathrm{C}:(s, t)=(0.0,-3.85) \times 10^{-3}$ (non-Skyrme point): $\quad M_{\mathrm{C}} \simeq 4.6 \mathrm{TeV}$.

The upper limit on the possible electroweak baryon mass in the event that the LHC does not find non-zero higher-order effective Lagrangian parameters is $\simeq 8 \mathrm{TeV}$, very similar to the bound quoted in [44]. This reflects the fact that the point with maximal mass overall is quite close to the Skyrme limit $t=0$. In this case, unlike the previous examples, the upper limit on the soliton mass is not relaxed by allowing a non-Skyrme term with $t<0$.

\section{Electroweak baryons as dark matter?}

If they exist, electroweak baryons should be present in the Universe today, and could provide cold dark matter [60]. Their possible relic density today depends on the presence and magnitude of a primordial electroweak baryon asymmetry. If this was small, and the electroweak baryon density was completely equilibriated in the early Universe, the present abundance of electroweak baryons would be insufficient to provide the present density of cold dark matter. In this case, one or more other sources of cold dark matter would be required, and the constraints on dark matter scattering that we now discuss would be irrelevant.

Assuming that they make up the bulk of the cold dark matter density, it is clear that electroweak baryons should have no electric charge. Moreover, it was pointed out in [64]

\footnotetext{
${ }^{4}$ Assuming that the model-dependent corrections to the classical mass calculation are not very large.
} 


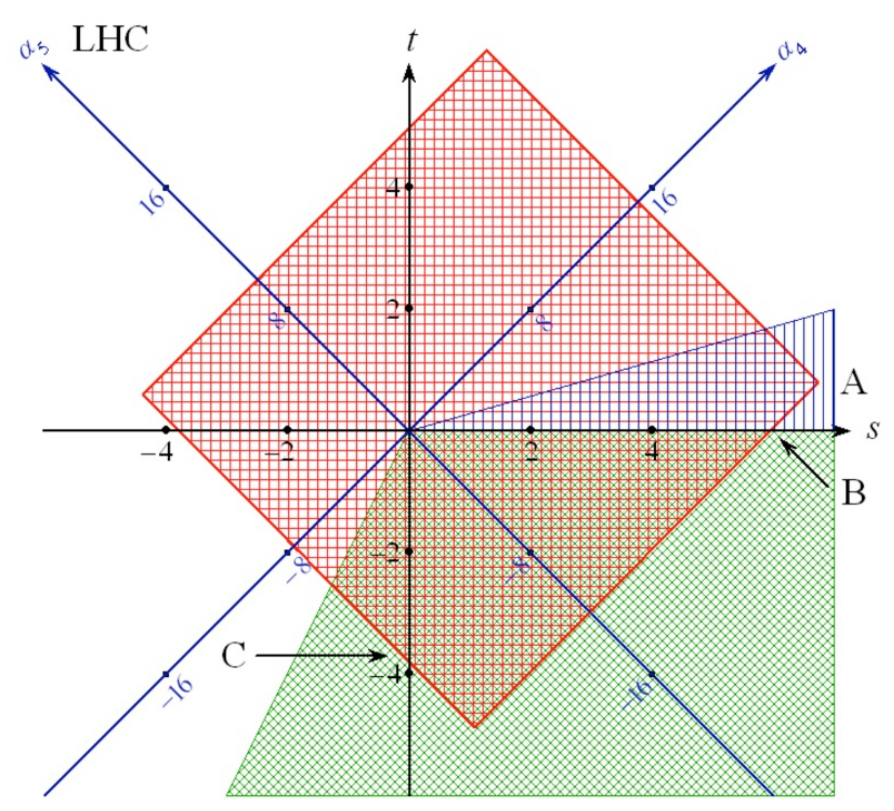

Figure 6. Comparison of the estimated LHC sensitivity range [58] for higher-order electroweak Lagrangian parameters with the the range of parameters where stable electroweak baryonic solitons exist. The parameters $\alpha_{4}, \alpha_{5}, s$ and $t$ are in units of $10^{-3}$. Point $\mathrm{A}$ has the maximal mass overall, Point $\mathrm{B}$ has the maximal mass allowed in the pure Skyrme limit: $t=0$, and Point $\mathrm{C}$ has the maximal mass allowed in the limit where the Skyrme term vanishes: $s=0$.

that they could not be fermions, as these would have an unacceptably large cross section for spin-independent scattering through magnetic moment couplings. Some scenarios for the effective low-energy electroweak Lagrangian in which the lightest electroweak baryon is a neutral boson have been enumerated in [65], in an analysis based on the Wess-ZuminoWitten term and the standard relation $Q_{\mathrm{em}}=I_{3}+Y$. The neutral boson scenarios found do not include $\mathrm{SU}(3) \times \mathrm{SU}(3) \rightarrow \mathrm{SU}(3)$ (which could yield a neutral fermion if $N_{\mathrm{c}}=3$ or a bosonic baryon if $N_{\mathrm{c}}$ is even), but $d o$ include $\mathrm{SU}(N) \times \mathrm{SU}(N) \rightarrow \mathrm{SU}(N)$ with $N>3$ and $N_{\mathrm{c}}$ even, and $\mathrm{SO}(N) \times \mathrm{SO}(N) \rightarrow \mathrm{SO}(N)$. However, $\mathrm{SU}(N) \rightarrow \mathrm{SO}(N)$ would yield a boson with charge $N_{\mathrm{c}}$, and the other coset structures considered in [65] have trivial homotopy and hence no electroweak baryons.

It should be noted that dark matter scattering is a non-trivial constraint, even if the lightest electroweak baryon is a neutral boson. The scattering cross section for this case was estimated in [61], with results indicating that models containing a pseudo-dilaton identified with the recently-discovered boson $X$ with mass $\simeq 125 \mathrm{GeV}$ would be consistent with the (updated) XENON100 limit [63] if the pseudo-dilaton couplings were scaled by $\geq 1 \mathrm{TeV} .{ }^{5}$ and the electroweak baryon weighed $\leq 1 \mathrm{TeV}$, as seen in figure 4 of [61]. The XENON100 Collaboration does not report results for larger dark matter particle masses, but naive extrapolation of the theoretical calculations and experimental sensitivity would suggest some tension for masses above $1 \mathrm{TeV}$.

\footnotetext{
${ }^{5}$ This is in the ball-park calculated in holographic dilaton models, and is reported to be consistent with the available data on $X(125)$ [40], once the contributions of the strongly-interacting electroweak sector the $X g g$ and $X \gamma \gamma$ couplings are taken into account.
} 
Our results suggest that scenarios which, according to the analysis of [65], might yield either a charged bosonic baryon or a fermionic baryon should not necessarily be abandoned. By the same token, one might not need to worry about the prospective tension with XENON100. This is because the parameters of the fourth-order effective Lagrangian might be in the range where no stable solitonic baryon exists, i.e., $t>0$ or $t<0$ and $t / s>2$. That said, it could be that stable electroweak baryons nevertheless exist for such parameter choices, but cannot be found using the solitonic Ansatz discussed here.

\section{Concluding remarks}

In this paper we have revisited the existence, stability and possible masses of classical solitonic solutions to the effective low-energy Lagrangians of QCD and a possible stronglyinteracting electroweak sector, with particular attention to the presence and consequences of a possible non-Skyrme quartic term as in $(2.4)[45-48,50]$. We have revisited the stability constraints in the $(s, t)$ plane, see figure 1 , and given general results for the classical soliton mass, see, e.g., figure 3.

In the QCD case, we have found that current phenomenological constraints on the quartic chiral Lagrangian terms $[56,57]$ allow a range of classical baryon masses that is somewhat broader than in the pure Skyrme case with $t=0$. In the case of a possible strongly-interacting electroweak sector, current data [58] allow a somewhat wider range of $t / s$ than in $\mathrm{QCD}$, and the expansion in the possible range of electroweak baryon masses is proportionally larger than in the pure Skyrme case. On the other hand, the prospective LHC sensitivity [58] to quartic Lagrangian parameters would not allow masses substantially larger than in the Skyrme limit.

It would be interesting to establish a 'no-lose' theorem that the LHC will either find non-zero higher-order Lagrangian parameters or discover electroweak baryons. However, this would require greater LHC sensitivity than indicated by the prospective range (3.7). To our knowledge, there is no accurate estimate of the range of electroweak baryon masses that the LHC could detect, but it is surely less than the ranges quoted above on the basis of a theoretical estimate of the possible LHC sensitivity to higher-order Lagrangian terms. On the other hand, the prospective LHC sensitivity should be revisited by a full experimental simulation including the possibility of high-luminosity LHC running.

As already commented, in order to be a valid dark matter candidate, a stable electroweak solitonic baryon should be a neutral boson. Even in this case, figure 4 of [61] indicates that such a model is quite constrained, at least in models in which the recentlydiscovered new boson is interpreted as a pseudo-dilaton. The estimate given in [61] of the spin-independent cold dark matter scattering cross section rises with the Skyrmion mass, so that experiments such as XENON100 [63] may be more sensitive to models with heavier electroweak baryons. Full exploration of this issue lies beyond the scope of this paper, but it is clear that dark matter scattering experiments are potentially interesting probes of strongly-interacting electroweak models with Skyrmion solutions. 


\section{Acknowledgments}

The work of J.E. was supported partly by the London Centre for Terauniverse Studies (LCTS), using funding from the European Research Council via the Advanced Investigator Grant 267352. The work of M.P. was partly supported by the Polish NCN grant 2011/01/B/ST2/00492.

Open Access. This article is distributed under the terms of the Creative Commons Attribution License which permits any use, distribution and reproduction in any medium, provided the original author(s) and source are credited.

\section{References}

[1] E. Witten, Current algebra, baryons and quark confinement, Nucl. Phys. B 223 (1983) 433 [INSPIRE].

[2] G.S. Adkins, C.R. Nappi and E. Witten, Static properties of nucleons in the Skyrme model, Nucl. Phys. B 228 (1983) 552 [inSPIRE].

[3] N. Seiberg and E. Witten, Electric-magnetic duality, monopole condensation and confinement in $N=2$ supersymmetric Yang-Mills theory, Nucl. Phys. B 426 (1994) 19 [Erratum ibid. B 430 (1994) 485] [hep-th/9407087] [INSPIRE].

[4] N. Seiberg and E. Witten, Monopoles, duality and chiral symmetry breaking in $N=2$ supersymmetric QCD, Nucl. Phys. B 431 (1994) 484 [hep-th/9408099] [INSPIRE].

[5] T. Skyrme, A nonlinear field theory, Proc. Roy. Soc. Lond. A 260 (1961) 127 [InSPIRE].

[6] T. Skyrme, A unified field theory of mesons and baryons, Nucl. Phys. 31 (1962) 556 [INSPIRE].

[7] G.S. Adkins and C.R. Nappi, The Skyrme model with pion masses, Nucl. Phys. B 233 (1984) 109 [INSPIRE].

[8] G.S. Adkins and C.R. Nappi, Model independent relations for baryons as solitons in mesonic theories, Nucl. Phys. B 249 (1985) 507 [INSPIRE].

[9] M. Karliner and M.P. Mattis, Hadron dynamics in the three flavor Skyrme model, Phys. Rev. Lett. 56 (1986) 428 [INSPIRE].

[10] M.P. Mattis and M. Karliner, The baryon spectrum of the Skyrme model, Phys. Rev. D 31 (1985) 2833 [INSPIRE].

[11] M. Karliner and M.P. Mattis, $\pi N, K N$ and $\bar{K} N$ scattering: Skyrme model versus experiment, Phys. Rev. D 34 (1986) 1991 [InSPIRE].

[12] U.G. Meissner, Low-energy hadron physics from effective chiral lagrangians with vector mesons, Phys. Rept. 161 (1988) 213 [INSPIRE].

[13] H. Weigel, Chiral soliton models for baryons, Lect. Notes Phys. 743 (2008) 1 [INSPIRE].

[14] S. Weinberg, Implications of dynamical symmetry breaking, Phys. Rev. D 13 (1976) 974 [INSPIRE].

[15] L. Susskind, Dynamics of spontaneous symmetry breaking in the Weinberg-Salam theory, Phys. Rev. D 20 (1979) 2619 [INSPIRE]. 
[16] ATLAS collaboration, Observation of a new particle in the search for the standard model Higgs boson with the ATLAS detector at the LHC, Phys. Lett. B 716 (2012) 1 [arXiv: 1207.7214] [INSPIRE].

[17] CMS collaboration, Observation of a new boson at a mass of $125 \mathrm{GeV}$ with the CMS experiment at the LHC, Phys. Lett. B 716 (2012) 30 [arXiv:1207.7235] [INSPIRE].

[18] G. Giudice, C. Grojean, A. Pomarol and R. Rattazzi, The strongly-interacting light Higgs, JHEP 06 (2007) 045 [hep-ph/0703164] [INSPIRE].

[19] R. Contino, C. Grojean, M. Moretti, F. Piccinini and R. Rattazzi, Strong double Higgs production at the LHC, JHEP 05 (2010) 089 [arXiv:1002.1011] [INSPIRE].

[20] R. Contino, The Higgs as a composite Nambu-Goldstone boson, arXiv:1005.4269 [INSPIRE].

[21] R. Grober and M. Muhlleitner, Composite Higgs boson pair production at the LHC, JHEP 06 (2011) 020 [arXiv: 1012.1562] [INSPIRE].

[22] B. Holdom, Techniodor, Phys. Lett. B 150 (1985) 301 [InSPIRE].

[23] K. Yamawaki, M. Bando and K.-i. Matumoto, Scale invariant technicolor model and a technidilaton, Phys. Rev. Lett. 56 (1986) 1335 [InSPIRE].

[24] T.W. Appelquist, D. Karabali and L. Wijewardhana, Chiral hierarchies and the flavor changing neutral current problem in technicolor, Phys. Rev. Lett. 57 (1986) 957 [INSPIRE].

[25] T. Akiba and T. Yanagida, Hierarchic chiral condensate, Phys. Lett. B 169 (1986) 432 [INSPIRE].

[26] M. Bando, K.-i. Matumoto and K. Yamawaki, Technidilaton, Phys. Lett. B 178 (1986) 308 [INSPIRE].

[27] W.D. Goldberger, B. Grinstein and W. Skiba, Distinguishing the Higgs boson from the dilaton at the Large Hadron Collider, Phys. Rev. Lett. 100 (2008) 111802 [arXiv:0708.1463] [INSPIRE].

[28] J. Fan, W.D. Goldberger, A. Ross and W. Skiba, Standard Model couplings and collider signatures of a light scalar, Phys. Rev. D 79 (2009) 035017 [arXiv:0803.2040] [INSPIRE].

[29] L. Vecchi, Phenomenology of a light scalar: the dilaton, Phys. Rev. D 82 (2010) 076009 [arXiv: 1002.1721] [INSPIRE].

[30] B. Grinstein and P. Uttayarat, A very light dilaton, JHEP 07 (2011) 038 [arXiv:1105.2370] [INSPIRE].

[31] S. Matsuzaki and K. Yamawaki, Techni-dilaton signatures at LHC, Prog. Theor. Phys. 127 (2012) 209 [arXiv: 1109.5448] [INSPIRE].

[32] V. Barger, M. Ishida and W.-Y. Keung, Dilaton at the LHC, Phys. Rev. D 85 (2012) 015024 [arXiv:1111.2580] [INSPIRE].

[33] B. Coleppa, T. Gregoire and H.E. Logan, Dilaton constraints and LHC prospects, Phys. Rev. D 85 (2012) 055001 [arXiv:1111.3276] [INSPIRE].

[34] C. Corianò, L. Delle Rose, A. Quintavalle and M. Serino, Effective dilaton interactions from the anomalous breaking of scale invariance of the standard model, arXiv:1206.0590 [INSPIRE].

[35] S. Matsuzaki and K. Yamawaki, Discovering 125 GeV techni-dilaton at LHC, Phys. Rev. D 86 (2012) 035025 [arXiv: 1206.6703] [INSPIRE]. 
[36] C. Corianò, L. Delle Rose, C. Marzo and M. Serino, Higher order dilaton interactions in the nearly conformal limit of the standard model, Phys. Lett. B 717 (2012) 182 [arXiv:1207.2930] [INSPIRE].

[37] S. Matsuzaki and K. Yamawaki, Is $125 \mathrm{GeV}$ techni-dilaton found at LHC?, Phys. Lett. B 719 (2013) 378 [arXiv:1207.5911] [INSPIRE].

[38] D. Elander and M. Piai, The decay constant of the holographic techni-dilaton and the $125 \mathrm{GeV}$ boson, arXiv: 1208.0546 [INSPIRE].

[39] L. Delle Rose and M. Serino, Dilaton interactions in $Q C D$ and in the electroweak sector of the standard model, AIP Conf. Proc. 1492 (2012) 210 [arXiv:1208.6432] [InSPIRE].

[40] S. Matsuzaki and K. Yamawaki, Holographic techni-dilaton at $125 \mathrm{GeV}$, Phys. Rev. D 86 (2012) 115004 [arXiv: 1209.2017] [INSPIRE].

[41] Z. Chacko and R.K. Mishra, Effective theory of a light dilaton, arXiv:1209.3022 [INSPIRE].

[42] Z. Chacko, R. Franceschini and R.K. Mishra, Resonance at 125 GeV: Higgs or dilaton/radion?, arXiv:1209.3259 [INSPIRE].

[43] B. Bellazzini, C. Csáki, J. Hubisz, J. Serra and J. Terning, A Higgs-like dilaton, arXiv:1209.3299 [INSPIRE].

[44] J. Ellis and M. Karliner, Indications on the mass of the lightest electroweak baryon, Phys. Lett. B 713 (2012) 233 [arXiv:1204.6642] [INSPIRE].

[45] D.E.L. Pottinger and E. Rathske, Metastability of solitons in a generalized Skyrme model, Phys. Rev. D 33 (1986) 2448 [INSPIRE].

[46] E. Rathske, Research on phenomenological models with chiral symmetry, BONN-IR-91-70 (1991) [INSPIRE].

[47] K. Fujii, S. Otsuki and F. Toyoda, Solitons with the Hopf index versus skyrmions in $\mathrm{SU}(2)$ nonlinear $\sigma$-model, Prog. Theor. Phys. 73 (1985) 1287 [INSPIRE].

[48] M. Lacombe, B. Loiseau, R. Vinh Mau and W. Cottingham, Nuclear binding and the Skyrme model, Phys. Lett. B 161 (1985) 31 [InSPIRE].

[49] J.F. Donoghue, E. Golowich and B.R. Holstein, Predicting the proton mass from $\pi \pi$ scattering data, Phys. Rev. Lett. 53 (1984) 747 [inSPIRE].

[50] A.A. Andrianov, V.A. Andrianov and V.Y. Novozhilov, Comment on 'Predicting the proton mass from $\pi \pi$ scattering data', Phys. Rev. Lett. 56 (1986) 1882 [INSPIRE].

[51] I. Aitchison, C. Fraser, E. Tudor and J. Zuk, Failure of the derivative expansion for studying stability of the baryon as a chiral soliton, Phys. Lett. B 165 (1985) 162 [INSPIRE].

[52] G.S. Adkins and C.R. Nappi, Stabilization of chiral solitons via vector mesons, Phys. Lett. B 137 (1984) 251 [INSPIRE].

[53] M. Mashaal, T.N. Pham and T.N. Truong, Can skyrmion be a good description of nucleon?, Phys. Rev. Lett. 56 (1986) 436 [INSPIRE].

[54] B. Moussallam, Casimir energy in the Skyrme model, Conf. Proc. C 9209271 (1992) 269 [hep-ph/9211229] [INSPIRE].

[55] G. Holzwarth and H. Walliser, Quantum corrections to the skyrmion mass, Nucl. Phys. A 587 (1995) 721 [INSPIRE]. 
[56] G. Ecker, J. Gasser, A. Pich and E. de Rafael, The role of resonances in chiral perturbation theory, Nucl. Phys. B 321 (1989) 311 [INSPIRE].

[57] A. Pich, Low-energy constants from resonance chiral theory, PoS (CONFINEMENT8) 026 [arXiv:0812.2631] [INSPIRE].

[58] O. Eboli, M. Gonzalez-Garcia and J. Mizukoshi, $p p \rightarrow j j e^{ \pm} \mu^{ \pm} \nu \nu$ and $j j e^{ \pm} m u^{\mp} \nu \nu$ at $O\left(\alpha_{e m}^{6}\right)$ and $O\left(\alpha_{e m}^{4} \alpha_{s}^{2}\right)$ for the study of the quartic electroweak gauge boson vertex at CERN LHC, Phys. Rev. D 74 (2006) 073005 [hep-ph/0606118] [INSPIRE].

[59] J. Distler, B. Grinstein, R.A. Porto and I.Z. Rothstein, Falsifying models of new physics via WW scattering, Phys. Rev. Lett. 98 (2007) 041601 [hep-ph/0604255] [INSPIRE].

[60] S. Nussinov, Technocosmology: could a technibaryon excess provide a 'natural' missing mass candidate?, Phys. Lett. B 165 (1985) 55 [InSPIRE].

[61] B.A. Campbell, J. Ellis and K.A. Olive, Phenomenology and cosmology of an electroweak pseudo-dilaton and electroweak baryons, JHEP 03 (2012) 026 [arXiv:1111.4495] [INSPIRE].

[62] T.N. Pham and T.N. Truong, Evaluation of the derivative quartic terms of the meson chiral lagrangian from forward dispersion relation, Phys. Rev. D 31 (1985) 3027 [INSPIRE].

[63] XENON100 collaboration, Dark matter results from 225 live days of XENON100 data, Phys. Rev. Lett. 109 (2012) 181301 [arXiv:1207.5988] [INSPIRE].

[64] J. Bagnasco, M. Dine and S.D. Thomas, Detecting technibaryon dark matter, Phys. Lett. B 320 (1994) 99 [hep-ph/9310290] [INSPIRE].

[65] M. Gillioz, Dangerous skyrmions in little Higgs models, JHEP 02 (2012) 121 [arXiv:1111.2047] [INSPIRE]. 